\title{
Foreword: mycotoxins in a changing world
}

This special issue arose because of the changes in the global landscape in relation to the impact and implications of our changing climate on food security and quality, consumer habits, trade and economics, regulations and scientific thinking. The EU green paper (EC, 2007) on climate change (CC) has suggested significant hot spots in different regions where food production will be considerably affected both in quality and quantity. Indeed, a recent UNEP report on 'Emerging Issues of Environmental Concern' (UNEP, 2016) has included a section entitled 'Poisoned chalice: Toxin accumulation in crops in an era of climate change' which refers to the impact that aflatoxin contamination is having in low and middle income countries (LMICs).

Recent studies suggest that CC will impact on cereal phenology with earlier flowering in wheat and maize and a shorter production window. Consequently, the geographical distribution of mycotoxigenic fungi, such as Aspergillus flavus, will be expanded into more northern and eastern regions of Europe. This could result in significantly higher contamination with aflatoxins and probability of large regions being non-compliant with EU legislative limits for food and feed (Battilani et al., 2016). It is possible that similar impacts could be felt in other important staple food production areas in Asia, North and South America. This will have both food security and economic effects.

Thus changes in the environment in which staple crops are grown as well as the predicted global movement of fungal diseases and pests and a change in their diversity are major drivers which will have significant implications for the predominant mycotoxins on a regional basis. Indeed, the impact of CC on mycotoxin contamination of key staple food/feed commodities will also have significant economic consequences, especially in developing countries.

In this special issue, topics were included presenting readers with a broad insight into the effects the changing world may have on mycotoxins, on a global scale, but also within specific fields of mycotoxin research. The latter aspects help to understand the role of different scientific disciplines as a whole to ensure, in a sustainable manner, safe and sufficient food and feed supplies.

We thus believe that this is a very timely special issue which addresses some of the key research areas with a mixture of review and primary papers. Authors were encouraged to speculate within the boundaries of their expert knowledge on the impacts that such changes might have on mycotoxins. Interdisciplinary approaches were stimulated to emphasise the importance of utilising the whole array of knowledge from molecular pathways for biosynthesis of mycotoxins to minimisation strategies, diagnostic tools to pre- and post-harvest predictive modelling and economic impacts.

This special issue of World Mycotoxin Journal includes a number of major themes which cover the breadth of research activities relevant to mycotoxin research, divided over various sections. The implications of changes in environment on shifts in the diversity of mycotoxigenic fungi, the agronomy of crops as drivers of changes in infection of cereals by mycotoxigenic fungi, including impacts at a fundamental molecular/functional level on mycotoxin production and on commodity productivity are considered.

The area of predictive modelling has been addressed which provides insights into the state of the art science on the impact of CC and the interaction between environmental factors and mycotoxin production. Will changes in agricultural practice and land use be required? The impacts of organic vs conventional cultivation issues and the benefits and challenges of biocontrol strategies are examined. Other very important issues are the consequences that environmental change might have on the economics and management of mycotoxins and the perceptions and food consumption habits of consumers who want to have residue-free food commodities. Will this require changes in the types of risk and exposure assessments, especially in relation to mixtures of mycotoxins? Furthermore, the large amount of data now becoming available on the so-called modified mycotoxins indicate that traditional analytical methods can underestimate the amount of relevant mycotoxins present in food and feed. These topics are all covered in this special issue. This provides a holistic view from multidisciplinary angles of the state of the art in 
mycotoxin research relevant to climate and environmental shifts which will impact globally on food safety and food security. Below we have summarised the key aspects which are covered in this special issue.

A key driver of mycotoxin research is the influence that mycotoxins have on economics and trade of major staple commodities especially between LMICs and developed countries. This is a key context for carrying out research which is relevant to the development of minimisation and prevention strategies. Wu and Mitchell (2016) have identified CC as the greatest factor affecting the mycotoxin problem. They have focused on existing and future scenarios of $\mathrm{CC}$ and how these will influence fungal infection and production of specific mycotoxins in food crops. The relevant impact of existing maximum allowable mycotoxin limits is discussed. A case study of how maize growers in the USA will be affected is included and the overall conclusions are that aflatoxins are the mycotoxins most likely to increase under future $\mathrm{CC}$ scenarios and which will have the greatest economic impact in the future. This is the context in which this special issue should be seen.

The $2^{\text {nd }}$ section, Environment, mycotoxins and predictive modelling, contains a series of four papers dealing with the effects that changing environments have at a genomic level, utilisation of these data for predicting production of mycotoxins and then two papers where a specific case of Fusarium graminearum and head blight of wheat is addressed followed by a holistic paper on predicting the production of mycotoxins using geographical and ecological data sets. Gilbert et al. (2016) examined the consequences that $\mathrm{CC}$ scenarios may have on growth and toxigenic potential of $A$. flavus. The application of functional genomics, including genomic sequencing, RNA-seq, microarray technologies and proteomics have revealed $\mathrm{CC}$-related effects on the aflatoxin cluster and plant-fungus interactions. Furthermore, elevated $\mathrm{CO}_{2}$ levels have been shown to impact on expression of the aflatoxin biosynthetic regulatory gene $a f l R$. The use of functional genomics will allow researchers to better understand the underlying transcriptomic response within the fungus to $\mathrm{CC}$, with a view towards predicting changes in fungal infection and toxin production under such changed environmental conditions. Magan and Medina (2016) have used microarrays to examine the relationship between interacting CC conditions on growth and toxin gene cluster expression. The relationship of these to phenotypic toxin production suggests that the effect on mycotoxin production is commonly much more significant as compared to the effect on growth. Modelling application to these data sets is suggested as a powerful tool for predicting the relative toxin production under extreme stress conditions, including $\mathrm{CC}$ scenarios. Different mycotoxigenic fungi may respond differently to $\mathrm{CC}$ conditions and thus extrapolation should be avoided or done with care. Vaughan et al.
(2016) examined the effect of changes mediated by future environments, especially climatically, on the evolutionary ecology of the F. graminearum species complex and future Fusarium Head Blight (FHB) epidemic patterns. A multitude of factors and key processes should be taken into account in order to anticipate the magnitude and direction of the change in FHB and mycotoxin risk, which may be challenging for quantitative assessments due to gaps in knowledge. It is suggested that simulation modelling approaches will be very helpful. Further research is needed to understand the combined influence of multiple abiotic factors on host defences, and the mechanisms by which individual and combined factors modulate wheat defences during Fusarium infection to optimise breeding efforts and climate resilient management strategies. Zhang et al. (2016) observed that both the Fusarium fujikuroi species complex (FFSC) and the F. graminearum species complex are the causal agents of Fusarium ear rot in the maize producing areas in China. Fumonisin $\mathrm{B}_{1}$-producing Fusarium verticillioides was the most prevalent species, followed by fumonisin $\mathrm{B}_{1}$-producing Fusarium proliferatum and 15-acetyldeoxynivalenol-producing F. graminearum. Both Fusarium temperatum and Fusarium boothii were identified for the first time in cooler regions in China. Mating type analysis and virulence tests showed adaptation to the host. The authors concluded that wheat/maize rotation selects for $F$. graminearum, while a wheat/rice rotation selects for Fusarium asiaticum. In contrast, F. boothii is selected when maize is cultivated without rotation. This shows that a change of crop - due to climate change, change in consumer preference or other economic reasons - can lead to a change in prevalent fungal species and type of mycotoxin produced. It might also lower the infection pressure of the replaced fungal species to other susceptible crops in that area. Van der Fels-Klerx et al. (2016) reviewed the available data of CC impacts on mycotoxin contamination in the field using a modelling approach. Only three examples have been published with a focus on Fusarium in wheat, $A$. flavus in maize and wheat and Alternaria in tomato. These have all been focused on Europe. The two main research gaps were identified regarding existing models. There has been very limited focus on some selected mycotoxin-crop combinations. Their validation has only been completed in one/few countries due to the (limited) availability and accessibility of data. Overall, the modelling approach shows some promise and should be integrated with models for other combinations of mycotoxins-crops to be able to better assist in mycotoxin management during crop cultivation in Europe, and to mitigate the expected CC impacts on mycotoxins.

With the rapid advances in the development of mycotoxin analytical systems there has been significant interest in the possible shifts in the occurrence of fungi and mycotoxins and whether biochemical transformations in the plant may be impacted by environmental changes. To this end 
two papers is the $3^{\text {rd }}$ section have addressed the area of Shifts in occurrence of mycotoxins, with a focus on masked mycotoxins. Dall'Asta and Battilani (2016) showed that recently an increasing number of studies on masked/ modified mycotoxins have been published. They have focused on fumonisins where knowledge of modified forms is still in its infancy because of the wide range of interactions the toxins may have with the matrix, and of the complexity of the $F$. verticillioides-Zea mays pathosystem. Extreme events, mentioned in CC scenarios may modify the dominant mycotoxigenic fungi and influence both mycotoxin production and their modification. The authors propose that modified fumonisins should be included in the monitoring plans to get an overview of their possible contribution to human exposure. Future research should include events occurring in the field and on the crosstalk between the plant and the fungus, to support the identification of resistant hybrids and to provide data for predictive models, the most suitable tool to forecast what is going to happen in the future changing climate. Lemmens et al. (2016) suggested that not only CC, but also contemporary developments in agriculture, such as breeding for enhanced FHB-resistance may result in changes in patterns of mycotoxins and their masked forms. For example, in new wheat varieties this is visible for deoxynivalenol (DON) and its modified form DON-3glucoside (DON-3G), where the most resistant lines with the lowest DON contamination show the highest relative level of DON-3G to DON. The authors hypothesise that a relatively higher proportion of $\mathrm{DON}$ is converted in planta to DON-3G in lines with an improved FHB resistance. The information provided here comes at a timely moment, since the CONTAM Panel of the European Food Safety Authority (EFSA) is currently in the process of drafting a Scientific Opinion about the risks to human and animal health of DON and its modified forms, including DON-3G.

Sustainable agricultural practices and precision systems have resulted in changes in Agricultural practices and land use. This issue, the subject area of the $4^{\text {th }}$ section, has been addressed in papers dealing with organic vs sustainable systems and the practical utilisation of biocontrol agents for aflatoxins in maize/groundnuts. Brodal et al. (2016) reviewed conventional and organic systems and the impact that they have on contamination of different commodities with mycotoxins. Data were from controlled field studies, farm surveys and some food basket surveys. Almost 50\% focused on DON in cereals. Other toxins looked at were HT-2/T-2 toxins, zearalenone and patulin. For the Fusarium toxins, levels in organically produced grains were either similar or (slightly) lower than in conventionally produced grains. Many studies suggest that weather condition, year of production, type of crop rotation and tillage practice have a greater impact on DON than whether conventional or organic agricultural practices were applied. For patulin in apples, higher contamination was found in organically than in conventionally produced apple/apple products. This review suggests that more systematic comparisons from scientifically controlled field trials and surveys are necessary to clarify whether differences in the risk of mycotoxin contamination between organically and conventionally produced crops are indeed real. Bandyopadhyay et al. (2016) indicated that contamination with aflatoxins is still a major concern; its occurrence transcends health, food security and trade sectors, affecting all actors in value chains and consumers. Use of biocontrol products with atoxigenic $A$. flavus active ingredients is a proven method for reducing the aflatoxin content of crops. In the USA, biopesticides based on atoxigenic $A$. flavus allow profitable crop production in areas that had previously experienced frequent aflatoxin contamination. The technology has been improved for use in sub-Saharan Africa, where efforts are underway to develop biocontrol products, under the trade name Aflasafe ${ }^{\mathrm{Tx}}$. The need for management programs that optimise both biocontrol's long-term and area-wide benefits is stressed in this paper. Benefits as well as potential apprehensions regarding this technology are discussed together with potential challenges, including the impact of CC scenarios on the production, formulation and efficacy of such biocontrol agents for aflatoxin minimisation.

The $5^{\text {th }}$ section in the special issue addresses the challenges in Risk assessment of multiple mycotoxins in food and consumer behaviour in relation to mycotoxins in a changing world. In this context, Assunção et al. (2016) emphasised that the risk of human co-exposure to multiple mycotoxins is real. There is growing concern about their potential impact on human health and regulations are not practically taking account of the risks of multiple exposure. Studies on hazard and exposure assessment of multiple mycotoxins, using harmonised methodologies, are crucial towards an improvement of data quality and a more reliable and robust risk characterisation, which is central for risk management and, consequently, to prevent mycotoxinsassociated adverse effects. An in-depth understanding of the nature of interactions between multiple mycotoxins will contribute to draw real conclusions on the health impact of human exposure to mycotoxin mixtures. Ragona (2016) showed that the trends in dietary compositions in our changing world can have a great impact on the exposure of populations to harmful substances in our food, including mycotoxins. Information about changes in available food and in consumer's choices may help authorities to decide whether exposure assessments need to be updated. The article discusses and analyses the factors contributing to the global trends in food consumption and the most prominent directions are identified in order to prioritise mycotoxin risk assessment. Unmistakably substantial changes in the availability of foods and in dietary patterns take place all over the world. An example shows how changing food available for consumption influences mycotoxin exposure. Perhaps not surprising is that the (scarce) information in 
the literature about awareness and perception of mycotoxin risks as compared to other foodborne hazards shows an inverse relation between education level and risk perception, an observation of relevance to risk communicators. De Nijs et al. (2016) examined five strategies to estimate mycotoxin exposure of a (sub-)population via food. This addresses the issues of data collection in the context of identifying the added values and limitations of each strategy for the estimation of chronic exposure to mycotoxins and, with the exception of the observed individual mean strategy, also acute exposure. Methods for data collection, i.e. food consumption surveys, food monitoring studies and total diet studies are discussed in detail. In particular human biomonitoring studies may also include exposure to mycotoxins from other sources than food, and exposure to modified mycotoxins that may not be detected with current analytical methods. Low limits of quantification are required for all analytical methods applied for data collection to avoid large uncertainties in the exposure due to high numbers of left censored data, i.e. with levels below the limit of quantification.

There is interest in the more routine use of Multi-mycotoxin analyses and this subject has been ably addressed in the $6^{\text {th }}$ section by Stroka and Maragos (2016). The rapid developments that have taken place in the analysis of mycotoxins include the application of techniques for the determination of multiple mycotoxins. (See the annual updates in World Mycotoxin Journal, e.g. Berthiller et al. (2016)). Although multi-mycotoxin methods offer great opportunities to get information about a broad spectrum of (groups of) mycotoxins in one analysis run, their use is accompanied with certain challenges. The need for co-extraction of multiple analytes with widely different polarities and the potential for carry-over of matrix components that can influence the results, are independent of the analytical techniques used (mass spectrometry or immunoassay). In addition there are specific challenges and opportunities that arise among the analytical platforms. Chromatographic methods for total aflatoxins could potentially be simplified to determine only one aflatoxin based on the use of stable response factors, which would reduce the analytical burden. Assays based on molecular recognition principles, such as immunoassays are currently no 'perfect' solution for multiplexed detection of mycotoxins. But these systems continue to be improved, eventually leading to fit-for-purpose methods.

In the last section of this special issue two additional papers are included dealing with related topics of CC impacts on fungi involved in the contamination of coffee and the impacts that may occur in relation to aflatoxins especially for LMICs. Thus Akbar et al. (2016) examined ochratoxigenic fungi and ochratoxin A (OTA) contamination of coffee. Aspergillus westerdijkiae strains were significantly impacted by CC and a stimulation of OTA production was observed. This was consistent with data available for A. flavus. However, for other species, especially Aspergillus carbonarius and Aspergillus niger, there was a reduction in OTA production under CC conditions. This suggests that different mycotoxigenic fungi may respond differently to $\mathrm{CC}$ conditions and thus extrapolation needs to be done with care. The possible impact of acclimatisation of the fungi to CC conditions needs to be examined in terms of whether this will influence colonisation rates of important commodities and whether this may lead to enhanced mycotoxin production. Wambui et al. (2016) focused on LMICs and how people living in developing countries may suffer more from mycotoxins in food and feed than those living in the industrialised part of the world. Indeed, a recent report by the IARC has demonstrated the impact that aflatoxins (and fumonisins) have on human health in these countries (Wild et al., 2015). Options exist, however, to mitigate this exposure. This paper has a special focus on Kenya, where cases of human aflatoxicosis have been recurrent, but its potential scope is much wider. CC is expected to lead to shifts from the current hot-spots to new hot-spots in Kenya. If no measures are taken, overall an increased part of the population is expected to be at risk. To protect the public and the environment from this negative impact, an integrated approach is proposed with management practices ranging from agronomic, postharvest through to institutional levels. The authors suggest a policy-oriented foresight to be developed in order to provide policy-based evidence for the applicability of the proposed measures.

We believe that the contents of this special issue confirm mycotoxins as a major challenge in a changing world. The impact of CC suggests that there may be complex interacting factors between the key abiotic stresses and plant physiology which makes predictions difficult without accurate data sets. We expect that in this context the research effort to address mycotoxins and their control will become even higher on the food safety and security agenda in the coming decades.

\section{Paola Battilani, Joerg Stroka and Naresh Magan}

Guest editors special issue 'Mycotoxins in a changing world'

Department of Sustainable Crop Production, Università Cattolica del Sacro Cuore, Piacenza, Italy; paola.battilani@unicatt.it

Joint Research Centre, IRMM, European Commission, Geel, Belgium; joerg.stroka@ec.europa.eu

Applied Mycology Group, Cranfield University, Cranfield, United Kingdom; n.magan@cranfield.ac.uk 


\section{References}

Akbar, A., Medina, A. and Magan, N., 2016. Impact of interacting climate change factors on growth and ochratoxin A production by Aspergillus section Circumdati and Nigri species on coffee. World Mycotoxin Journal 9: 863-874.

Assunção, R., Silva, M.J. and Alvito, P., 2016. Challenges in risk assessment of multiple mycotoxins in food. World Mycotoxin Journal 9: 791-811.

Bandyopadhyay, R., Ortega-Beltran, A., Akande, A., Mutegi, C., Atehnkeng, J., Kaptoge, L., Senghor, A.L., Adhikari, B.N. and Cotty, P.J., 2016. Biological control of aflatoxins in Africa: current status and potential challenges in the face of climate change. World Mycotoxin Journal 9: 771-789.

Battilani, P., Toscano, P., Van der Fels-Klerx, H.J., Moretti, A., Camardo Leggieri, M., Brera, C., Rortais, A., Goumperis, T. and Robinson, T., 2016. Aflatoxin $B_{1}$ contamination in maize in Europe increases due to climate change. Scientific Reports 6: 24328.

Berthiller, F., Brera, C., Crews, C., Iha, M.H., Krska, R., Lattanzio, V.M.T., McDonald, S., Malone, R.J., Maragos, C., Solfrizzo, M., Stroka, J. and Whitaker, T.B., 2016. Developments in mycotoxin analysis: an update for 2014-2015. World Mycotoxin Journal 9: 5-30.

Brodal, G., Hofgaard, I.S., Eriksen, G.S., Bernhoft, A. and Sundheim, L., 2016. Mycotoxins in organically versus conventionally produced cereal grains and some other crops in temperate regions. World Mycotoxin Journal 9: 755-770.

Dall'Asta, C. and Battilani, P., 2016. Fumonisins and their modified forms, a matter of concern in future scenario? World Mycotoxin Journal 9: 727-739.

De Nijs, M., Mengelers, M.J.B., Boon, P.E., Heyndrickx, E., Hoogenboom, L.A.P., Lopez, P. and Mol, H.G.J., 2016. Strategies for estimating human exposure to mycotoxins via food. World Mycotoxin Journal 9: 831-845.

European Commission (EC), 2007. Green paper from the Commission to the Council, the European Parliament, the European Economic and Social Committee and the Committee of the Regions. Adapting to climate change in Europe - options for EU action \{SEC(2007) 849\}. European Commission, Brussels, Belgium. Available at: http:// tinyurl.com/ohfgj3t.

Gilbert, M.K., Mack, B.M., Payne, G.A. and Bhatnagar, D., 2016. Use of functional genomics to assess the climate change impact on Aspergillus flavus and aflatoxin production. World Mycotoxin Journal 9: 665-672.
Lemmens, M., Steiner, B., Sulyok, M., Nicholson, P., Mesterhazy, A. and Buerstmayr, H., 2016. Masked mycotoxins: does breeding for enhanced Fusarium Head Blight resistance result in more deoxynivalenol-3-glucoside in new wheat varieties? World Mycotoxin Journal 9: 741-754.

Magan, N. and Medina, A., 2016. Integrating gene expression, ecology and mycotoxin production by Fusarium and Aspergillus species in relation to interacting environmental factors. World Mycotoxin Journal 9: 673-684.

Ragona, M., 2016. Mycotoxins, the unknowns: trends in food availability and consumer perceptions. World Mycotoxin Journal 9: 813-830.

Stroka, J. and Maragos, C.M., 2016. Challenges in the analysis of multiple mycotoxins. World Mycotoxin Journal 9: 847-861.

United Nations Environment Programme (UNEP), 2016. UNEP Frontier 2016 Report. Emerging issues of environmental concern. UNEP, Nairobi, Kenya.

Van der Fels-Klerx, H.J., Liu, C. and Battilani, P., 2016. Modelling climate change impacts on mycotoxin contamination. World Mycotoxin Journal 9: 717-726.

Vaughan, M., Backhouse, D. and Del Ponte, E.M., 2016. Climate change impacts on the ecology of Fusarium graminearum species complex and susceptibility of wheat to Fusarium Head Blight: a review. World Mycotoxin Journal 9: 685-700.

Wambui, J.M., Karuri, E.G., Ojiambo, J.A. and Njage, P.M.K., 2016. Adaptation and mitigation options to manage aflatoxin contamination in food with a climate change perspective. World Mycotoxin Journal 9: 875-888.

Wild, C.P., Miller, J.D. and Groopman, J.D., 2015. Mycotoxin control in low- and middle income countries. IARC Working Group Report No. 9. International Agency for Research on Cancer, Lyon, France.

Wu, F. and Mitchell, N.J., 2016. How climate change and regulations can affect the economics of mycotoxins. World Mycotoxin Journal 9: 653-663.

Zhang, H., Brankovics, B., Luo, W., Xu, J., Xu, J.S., Guo, C., Guo, J.G., Jin, S.L., Chen, W.Q., Feng, J., Van Diepeningen, A.D., Van der Lee, T.A.J. and Waalwijk, C., 2016. Crops are a main driver for species diversity and the toxigenic potential of Fusarium isolates in maize ears in China. World Mycotoxin Journal 9: 701-715. 
